\title{
Interval neutrosophic finite switchboard state machine
}

\author{
Tahir Mahmood $^{1}$ • Qaisar Khan ${ }^{1}$
}

\begin{abstract}
In this paper we introduced the concept of interval neutrosophic finite state machine, interval neutrosophic finite switchboard state machine using the notion of interval neutrosophic set. We also introduced the concept of homomorphism and strong homomorphism of interval neutrosophic finite state machine.
\end{abstract}

Keywords Interval neutrosophic set · Interval neutrosophic finite state machine $\cdot$ Interval neutrosophic finite switchboard state machine

Mathematics Subject Classification $\quad 03 \mathrm{D} 05 \cdot 20 \mathrm{M} 35 \cdot 68 \mathrm{Q} 70 \cdot 18 \mathrm{~B} 20 \cdot 68 \mathrm{Q} 45$

\section{Introduction}

The theory of fuzzy sets was introduced by Zadeh in 1965 [17] as a genralization of crisp sets. After the introduction of fuzzy sets many researcher applied the concept of fuzzy sets in various fields and achieved a great success. After that Zadeh made an extension of fuzzy sets and named this extension interval valued fuzzy set [18]. After these two extensions Attanasov introduced the concept of intutionistic fuzzy sets in 1986 [1]. That is representing objects by a membership and non membership fucntions. There were also other geralizations of fuzzy sets such as bipolar valued fuzzy set [8], vague sets [3], cubic sets [6], interval valued intutionistic fuzzy sets [2]. These were mathematical tools to discribe the uncertainity. Florentin Smarandache [12,13] introduced the concept of neutrosophy and neutrosophic sets which was the genralization of fuzzy sets, intuitionistic fuzzy sets, interval valued fuzzy set and al defined extensions, defined bove. Te word "heutrosophy" etymologically,

Qaisar Khan

qaisarkhan421@gmail.com

Tahir Mahmood

tahirbakhat@yahoo.com

1 Department of Mathematics and Statistics, International Islamic University, Islamabad, Pakistan 
"neutro-sophy" (noun) comes from French neutre Latin neuter, neutral, and Greek sophia, skill/wisdom means knowledge of neutral thought. Neutrosophy is a branch of philospy introduced by which studies the origin and scope of neutralities, as well as thier interaction with ideational spectra. This theory considers every notion or idea $\langle\mathrm{A}\rangle$ together with its opposite or negation $\langle$ antiA $\rangle$ and with their spectrum of neutralities $\langle$ neutA $\rangle$ in between them (i.e. notions or ideas supporting neither $\langle\mathrm{A}\rangle$ nor $\langle\operatorname{antiA}\rangle)$. The $\langle$ neut $\mathrm{A}\rangle$ and $\langle$ antiA $\rangle$ ideas together are referred to as $\langle$ non $A\rangle$. Neutrosophy is a generalization of Hegel's dialectics (the last one is based on $\langle\mathrm{A}\rangle$ and $\langle$ anti $\mathrm{A}\rangle$ only). While a "neutrosophic" (adjective), means having the nature of, or having the characteristic of Neutrosophy. A.neutrosophic set $A$ is charaterized by a truth membership function $T_{A}$, Indeterminancy membership function $I_{A}$, Falsity membership function $F_{A}$. Where $T_{A}, I_{A}$ and $F_{A}$ are real standard and nonstandard subsets of $]^{-} 0,1^{+}$. The neutrosophic sets is suitable for real life prolem, but it is difficult to apply in scientific problems. The difference between neutrosophic sets and intuitionistic fuzzy sets is that in neutrosophic sets the degree of indeterminancy is defined independently. To apply neutrosophic set in real life and in scientific problems Wang et al. defined single valued neutrosophic set and their set theoretic operators in 2011 [16]. In single valued neutrosophic set closed interval $[0,1]$ can be taken instead of $]^{-} 0,1^{+}[$. In 2005 Wang et al. definend interval neutrosophic set and thier set theoretic properties, convexcity, truth-favorite and falsity favorite interval neutrosophic set [15]. Malik et al. introduced the concept of submachine of fuzzy finite state machin, product of fuzzy finite state machine [10,11]. Malik et al. also introduced subsystem of fuzzy finite state machine [9]. In 2002 Kumbhojkar and Chaudhari introduced covering of fuzzy finite state machine [7]. Sato and Kuroki introduced fuzzy finite switchboard state machine in 2002 [14]. After the introduction of fuzzy finite state machine Jun in 2005 introduced the concept of intuitionistic fuzzy finite state state machine, intutionistic submachine and their related propertis were discussed [4]. In 2006 Jun introduced the concept of intuitionistic fuzzy finite switchboard state machine, commutative intuitionistic fuzzy finite state machine and strong homomorphism [5]. In this paper we introduced the concept of interval neutrosophic finite state machine, interval neutrosophic finite switchboard state machine using the notion of interval neutrosophic set. We also introduced the concept of homomorphism and strong homomorphism of interval neutrosophic finite state machine.

\section{Preliminaries}

In this section we define some basic definitions about intuitionistic fuzzy set, interval neutrosophic set and intuitionistic fuzzy finite state machine, intuitionistic fuzzy finite switchboard state machine defined in $[1,4,5,15]$.

Definition 2.1 [1] An intutionistic fuzzy set on the universal set $X$ is an object of the form

$$
H=\left\{\left\langle a, \mu_{H}(a), v_{H}(a)\right\rangle \mid a \in X\right\}
$$

where $\mu_{H}: X \rightarrow[0,1]$ and $v_{H}: X \rightarrow[0,1]$ are called the membership and non-membership functions respectively and the conditon that $0 \leq \mu_{H}(a)+v_{H}(a) \leq 1$ for all $a \in X$.

Definition 2.2 [4] A triple $M=(N, U, H)$ is called intuitionistic fuzzy finite state machine. In which $N, U, H$ are respectively, representing the set of states, the set of input symbols and intuitionistic fuzzy sets in $N \times U \times N$. 
Definition 2.3 [5] An intuitionistic fuzzy finite state machine $M=(N, U, H)$ is said to be switching if it satisfies:

$$
\mu_{H}(r, a, s)=\mu_{H}(s, a, r) \text { and } v_{H}(r, a, s)=v_{H}(s, a, r)
$$

for all $r, s, \in N$ and $a \in U$.

An intutionistic fuzzy finite state machine $M=(N, U, H)$ is said to be commutative if it satisfies:

$$
\mu_{H}(r, a b, s)=\mu_{H}(r, b a, s) \quad \text { and } v_{H}(r, a b, s)=v_{H}(r, b a, s)
$$

for all $r, s, \in N$ and $a, b \in U$.

An intutionistic fuzzy finite state machine $M=(N, U, H)$ which is both switching and commutative is called an intutionistic fuzzy finite switchboard state machine.

Definition 2.4 [15] Let $X$ be a universal set. An interval neutrosophic set (INS for short) is of the form

$$
\begin{aligned}
S & =\left\{\left\langle\alpha_{S}(a), \beta_{S}(a), \gamma_{S}(a)\right\rangle \mid a \in X\right\} \\
& =\left\{\left\langle a,\left[\inf \alpha_{S}(a), \sup \alpha_{S}(a)\right],\left[\inf \beta_{S}(a), \sup \beta_{S}(a)\right],\left[\inf \gamma_{S}(a), \sup \gamma_{S}(a)\right]\right\rangle \mid a \in X\right\} .
\end{aligned}
$$

where $\alpha_{S}(a), \beta_{S}(a)$ and $\gamma_{S}(a)$ respectively representing the truth-membership, indeterminancy-memebership and falsity membership functions for each $a \in X, \alpha_{S}(a)$, $\beta_{S}(a), \gamma_{S}(a) \subseteq[0,1]$ and the condition that $0 \leq \sup \alpha_{S}(a)+\sup \beta_{S}(a)+\sup \gamma_{S}(a) \leq 3$.

Definition 2.5 [15] An INS $S$ set is empty if $\inf \alpha_{S}(a)=\sup \alpha_{S}(a)=0, \inf \beta_{S}(a)=$ $\sup \beta_{S}(a)=1, \inf \gamma_{S}(a)=\sup \gamma_{S}(a)=1$ for all $a \in X$.

Definition 2.6 [15] Let $A$ and $B$ be two INSs. Then $A$ is contained in $B$ if and only if $\inf \alpha_{A}(a) \leq \inf \alpha_{B}(a), \sup \alpha_{A}(a) \leq \sup \alpha_{B}(a), \inf \beta_{A}(a) \geq \inf \beta_{B}(a), \sup \beta_{A}(a) \geq$ $\sup \beta_{B}(a), \inf \gamma_{A}(a) \geq \inf \gamma_{B}(a), \sup \gamma_{A}(a) \geq \sup \gamma_{B}(a)$.

\section{Interval neutrosophic finite state machine}

Definition 3.1 A triple $M=(N, U, S)$ is called interval neutrosophic finite state machine (INFSM for short), where $N, U$ are finite non-empty sets, called the set of states and input symbols respectively, and $S=\left\langle\alpha_{S}(a), \beta_{S}(a), \gamma_{S}(a)\right\rangle$ is an INS in $N \times U \times N$.

The set of all words of finite length of $U$ is denoted by $U^{*}$. The empty word is denoted by $\zeta$, and the length of each $a \in U^{*}$ is denoted by $|a|$.

Definition 3.2 Let $M=(N, U, S)$ be an INFSM. Define an INS $S^{*}=\left\langle\alpha_{S^{*}}(a), \beta_{S^{*}}(a)\right.$, $\left.\gamma_{S^{*}}(a)\right\rangle$ in $N \times U^{*} \times N$ by

$$
\begin{aligned}
& \alpha_{S^{*}}(r, \zeta, s):= \begin{cases}{[1,1]} & \text { if } r=s \\
{[0,0]} & \text { if } r \neq s\end{cases} \\
& \beta_{S^{*}}(r, \zeta, s):= \begin{cases}{[0,0]} & \text { if } r=s \\
{[1,1]} & \text { if } r \neq s\end{cases}
\end{aligned}
$$


and

$$
\begin{aligned}
\gamma_{S^{*}}(r, \zeta, s) & := \begin{cases}{[0,0]} & \text { if } r=s \\
{[1,1]} & \text { if } r \neq s\end{cases} \\
\alpha_{S^{*}}(r, a b, s) & =\vee_{v \in N}\left[\alpha_{S^{*}}(r, a, v) \wedge \alpha_{S}(v, b, s)\right], \\
\beta_{S^{*}}(r, a b, s) & =\wedge_{v \in N}\left[\beta_{S^{*}}(r, a, v) \vee \beta_{S}(v, b, s)\right]
\end{aligned}
$$

and

$$
\gamma_{S^{*}}(r, a b, s)=\wedge_{v \in N}\left[\gamma_{S^{*}}(r, a, v) \vee \gamma_{S}(v, b, s)\right]
$$

for all $r, s \in N, a \in U^{*}$ and $b \in U$.

Example 3.3 Let $N=\{r, s, v\}$ and $U=\{a, b\}$ and $S$ be INS defined by

$$
\begin{aligned}
& \alpha_{S}(r, a, v)=[0.1,0.2], \quad \beta_{S}(r, a, v)=[0.4,0.5], \quad \gamma_{S}(r, a, v)=[0.5,0.6] \\
& \alpha_{S}(r, b, s)=[0.5,0.7], \quad \beta_{S}(r, b, s)=[0.2,0.3], \quad \gamma_{S}(r, b, s)=[0.3,0.4] \\
& \alpha_{S}(s, a, v)=[0.2, o .3], \quad \beta_{S}(s, a, v)=[0.3,0.4], \quad \gamma_{S}(s, a, v)=[0.6,0.7] \\
& \alpha_{S}(v, a, v)=[0.3,0.4], \quad \beta_{S}(v, a, v)=[0.35,0.4], \quad \gamma_{S}(v, a, v)=[0.4,0.5] \\
& \alpha_{S}(v, b, s)=[0.8,0.9], \quad \beta_{S}(v, b, s)=[0,0], \quad \gamma_{S}(v, b, s)=[0.1,0.2] .
\end{aligned}
$$

Then $(N, U, S)$ is an INFSM.

The transition daigram is given below:

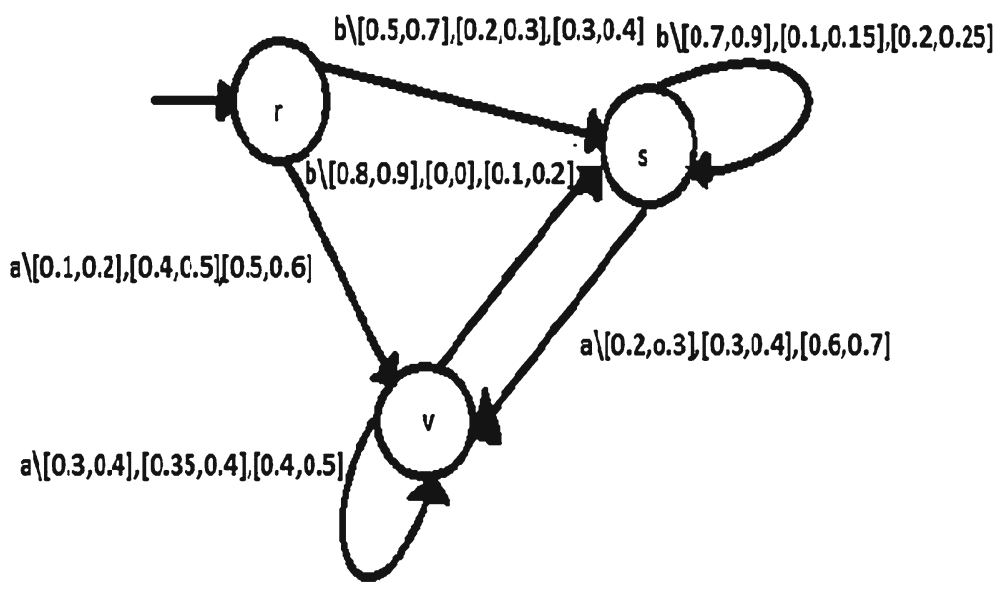

Lemma 3.4 Let $M=(N, U, S)$ be an INFSM. Then

$$
\begin{aligned}
\alpha_{S^{*}}(r, a b, s) & =\vee_{v \in N}\left[\alpha_{S^{*}}(r, a, v) \wedge \alpha_{S^{*}}(v, b, s)\right] \\
\beta_{S^{*}}(r, a b, s) & =\wedge_{v \in N}\left[\beta_{S^{*}}(r, a, v) \vee \beta_{S^{*}}(v, b, s)\right]
\end{aligned}
$$

and

$$
\gamma_{S^{*}}(r, a b, s)=\wedge_{v \in N}\left[\gamma_{S^{*}}(r, a, v) \vee \gamma_{S^{*}}(v, b, s)\right]
$$

for all $r, s \in N$ and $a, b \in U^{*}$. 
Proof Let $r, s \in N$ and $a, b \in U^{*}$. We prove the result by induction on $|b|=k$. If $k=0$, then $b=\zeta$, and so $a b=a \zeta=a$. Hence

$$
\begin{aligned}
\vee_{v \in N}\left[\alpha_{S^{*}}(r, a, v) \wedge \alpha_{S^{*}}(v, b, s)\right] & =\vee_{v \in N}\left[\alpha_{S^{*}}(r, a, v) \wedge \alpha_{S^{*}}(v, \zeta, s)\right] \\
& =\alpha_{S^{*}}(r, a, s)=\alpha_{S^{*}}(r, a b, s), \\
\wedge_{v \in N}\left[\beta_{S^{*}}(r, a, v) \vee \beta_{S^{*}}(v, b, s)\right] & =\wedge_{v \in N}\left[\beta_{S^{*}}(r, a, v) \vee \beta_{S^{*}}(v, \zeta, s)\right] \\
& =\beta_{S^{*}}(r, a, s)=\beta_{S^{*}}(r, a b, s)
\end{aligned}
$$

and

$$
\begin{aligned}
\wedge_{v \in N}\left[\gamma_{S^{*}}(r, a, v) \vee \gamma_{S^{*}}(v, b, s)\right] & =\wedge_{v \in N}\left[\gamma_{S^{*}}(r, a, v) \vee \gamma_{S^{*}}(v, \zeta, s)\right] \\
& =\gamma_{S^{*}}(r, a, s)=\gamma_{S^{*}}(r, a b, s) .
\end{aligned}
$$

So the result is true for $k=0$. Suppose that the result is true for $|c|=k-1$. That is for all $c \in U^{*}$ such that $|c|=k-1, k>0$. Let $b=c d$, where $c \in U^{*}$ and $d \in U$, and $|c|=k-1$. Then

$$
\begin{aligned}
\alpha_{S^{*}}(r, a b, s) & =\alpha_{S^{*}}(r, a c d, s)=\vee_{v \in N}\left[\alpha_{S^{*}}(r, a c, v) \wedge \alpha_{S}(v, d, s)\right] \\
& =\vee_{v \in N}\left[\vee_{w \in N}\left[\alpha_{S^{*}}(r, a, w) \wedge \alpha_{S^{*}}(w, c, v)\right] \wedge \alpha_{S}(v, d, s)\right] \\
& =\vee_{v \in N}\left[\vee_{w \in N}\left[\alpha_{S^{*}}(r, a, w) \wedge \alpha_{S^{*}}(w, c, v)\right] \wedge \alpha_{S}(v, d, s)\right] \\
& =\vee_{v, w \in N}\left[\alpha_{S^{*}}(r, a, w) \wedge \alpha_{S^{*}}(w, c, v) \wedge \alpha_{S}(v, d, s)\right] \\
& =\vee_{w \in N}\left[\alpha_{S^{*}}(r, a, w)\left(\vee_{v \in N}\left[\alpha_{S^{*}}(w, c, v) \wedge \alpha_{S}(v, d, s)\right)\right]\right. \\
& =\vee_{w \in N}\left[\alpha_{S^{*}}(r, a, w) \wedge \alpha_{S^{*}}(w, c d, s)\right] \\
& =\vee_{w \in N}\left[\alpha_{S^{*}}(r, a, w) \wedge \alpha_{S^{*}}(w, b, s)\right], \\
\beta_{S^{*}}(r, a b, s) & =\beta_{S^{*}}(r, a c d, s)=\wedge_{v \in N}\left[\beta_{S^{*}}(r, a c, v) \vee \beta_{S}(v, d, s)\right] \\
& =\wedge_{v \in N}\left[\wedge_{w \in N}\left[\beta_{S^{*}}(r, a, w) \vee \beta_{S^{*}}(w, c, v)\right] \vee \beta_{S}(v, d, s)\right] \\
& =\wedge_{v, w \in N}\left[\beta_{S^{*}}(r, a, w) \vee \beta_{S^{*}}(w, c, v) \vee \beta_{S}(v, d, s)\right] \\
& =\wedge_{w \in N}\left[\beta_{S^{*}}(r, a, w)\left(\wedge_{v \in N}\left[\beta_{S^{*}}(w, c, v) \vee \beta_{S}(v, d, s)\right)\right]\right. \\
& =\wedge_{w \in N}\left[\beta_{S^{*}}(r, a, w) \vee \beta_{S^{*}}(w, c d, s)\right] \\
& =\wedge_{w \in N}\left[\beta_{S^{*}}(r, a, w) \vee \beta_{S^{*}}(w, b, s)\right]
\end{aligned}
$$

and

$$
\begin{aligned}
\gamma_{S^{*}}(r, a b, s) & =\gamma_{S^{*}}(r, a c d, s)=\wedge_{v \in N}\left[\gamma_{S^{*}}(r, a c, v) \vee \gamma_{S}(v, d, s)\right] \\
& =\wedge_{v \in N}\left[\wedge_{w \in N}\left[\gamma_{S^{*}}(r, a, w) \vee \gamma_{S^{*}}(w, c, v)\right] \vee \gamma_{S}(v, d, s)\right] \\
& =\wedge_{v, w \in N}\left[\gamma_{S^{*}}(r, a, w) \vee \gamma_{S^{*}}(w, c, v) \vee \gamma_{S}(v, d, s)\right] \\
& =\wedge_{w \in N}\left[\gamma_{S^{*}}(r, a, w)\left(\wedge_{v \in N}\left[\gamma_{S^{*}}(w, c, v) \vee \gamma_{S}(v, d, s)\right)\right]\right. \\
& =\wedge_{w \in N}\left[\gamma_{S^{*}}(r, a, w) \vee \gamma_{S^{*}}(w, c d, s)\right] \\
& =\wedge_{w \in N}\left[\gamma_{S^{*}}(r, a, w) \vee \gamma_{S^{*}}(w, b, s)\right] .
\end{aligned}
$$

Therfore the result is true for $|b|=n$. This completes the proof.

\section{Interval neutrosophic finite switchboard state machine}

Definition 4.1 An INFSM $M=(N, U, S)$ is said to be switching if it satisfies:

$$
\alpha_{S}(r, a, s)=\alpha_{S}(s, a, r), \beta_{S}(r, a, s)=\beta_{S}(s, a, r)
$$


and

$$
\gamma_{S}(r, a, s)=\gamma_{S}(s, a, r)
$$

for all $r, s \in N$ and $a \in U$.

An INFSM $M=(N, U, S)$ is said to be commutative if it satisfies:

$$
\alpha_{S}(r, a b, s)=\alpha_{S}(r, b a, s), \beta_{S}(r, a b, s)=\beta_{S}(r, b a, s)
$$

and

$$
\gamma_{S}(r, a b, s)=\gamma_{S}(r, b a, s)
$$

for all $r, s \in N$ and $a, b \in U$.

If an INFSM $M=(N, U, S)$ is both switching and commutative, then it is called interval neutrosophic finite switchboard state machine (INFSSM for short).

Proposition 4.2 If $M=(N, U, S)$ is a commutative INFSM, then

$$
\alpha_{S^{*}}(r, b a, s)=\alpha_{S^{*}}(r, a b, s), \beta_{S^{*}}(r, b a, s)=\beta_{S^{*}}(r, a b, s)
$$

and

$$
\gamma_{S^{*}}(r, b a, s)=\gamma_{S^{*}}(r, a b, s) .
$$

for all $r, s \in N$ and $a \in U, b \in U^{*}$.

Proof Let $r, s \in N$ and $a, b \in U^{*}$. We prove the result by induction on $|b|=k$. If $k=0$, then $b=\zeta$, hence

$$
\begin{aligned}
& \alpha_{S^{*}}(r, b a, s)=\alpha_{S^{*}}(r, \zeta a, s)=\alpha_{S^{*}}(r, a, s)=\alpha_{S^{*}}(r, a \zeta, s)=\alpha_{S^{*}}(r, a b, s), \\
& \beta_{S^{*}}(r, b a, s)=\beta_{S^{*}}(r, \zeta a, s)=\beta_{S^{*}}(r, a, s)=\beta_{S^{*}}(r, a \zeta, s)=\beta_{S^{*}}(r, a b, s)
\end{aligned}
$$

and

$$
\gamma_{S^{*}}(r, b a, s)=\gamma_{S^{*}}(r, \zeta a, s)=\gamma_{S^{*}}(r, a, s)=\gamma_{S^{*}}(r, a \zeta, s)=\gamma_{S^{*}}(r, a b, s) .
$$

Therefore the result is true for $k=0$. Suppose that the result is true for $|c|=k-1$. That is for all $c \in U^{*}$ with $|c|=k-1, k>0$. Let $d \in U$ be such that $b=c d$. Then

$$
\begin{aligned}
\alpha_{S^{*}}(r, b a, s) & =\alpha_{S^{*}}(r, c d a, s)=\vee_{v \in N}\left[\alpha_{S^{*}}(r, c, v) \wedge \alpha_{S^{*}}(v, d a, s)\right] \\
& =\vee_{v \in N}\left[\alpha_{S^{*}}(r, c, v) \wedge \alpha_{S^{*}}(v, a d, s)\right] \\
& =\alpha_{S^{*}}(r, c a d, s) \\
& =\vee_{v \in N}\left[\alpha_{S^{*}}(r, c a, v) \wedge \alpha_{S}(v, d, s)\right] \\
& =\vee_{v \in N}\left[\alpha_{S^{*}}(r, a c, v) \wedge \alpha_{S}(v, d, s)\right] \\
& =\alpha_{S^{*}}(r, a c d, s)=\alpha_{S^{*}}(r, a b, s), \\
\beta_{S^{*}}(r, b a, s) & =\beta_{S^{*}}(r, c d a, s)=\wedge_{v \in N}\left[\beta_{S^{*}}(r, c, v) \vee \beta_{S^{*}}(v, d a, s)\right] \\
& =\wedge_{v \in N}\left[\beta_{S^{*}}(r, c, v) \vee \beta_{S^{*}}(v, a d, s)\right] \\
& =\beta_{S^{*}}(r, c a d, s) \\
& =\wedge_{v \in N}\left[\beta_{S^{*}}(r, c a, v) \vee \beta_{S}(v, d, s)\right] \\
& =\wedge_{v \in N}\left[\beta_{S^{*}}(r, a c, v) \vee \beta_{S}(v, d, s)\right] \\
& =\beta_{S^{*}}(r, a c d, s)=\beta_{S^{*}}(r, a b, s)
\end{aligned}
$$


and

$$
\begin{aligned}
\gamma_{S^{*}}(r, b a, s) & =\gamma_{S^{*}}(r, c d a, s)=\wedge_{v \in N}\left[\gamma_{S^{*}}(r, c, v) \vee \gamma_{S^{*}}(v, d a, s)\right] \\
& =\wedge_{v \in N}\left[\gamma_{S^{*}}(r, c, v) \vee \gamma_{S^{*}}(v, a d, s)\right] \\
& =\gamma_{S^{*}}(r, c a d, s) \\
& =\wedge_{v \in N}\left[\gamma_{S^{*}}(r, c a, v) \vee \gamma_{S}(v, d, s)\right] \\
& =\wedge_{v \in N}\left[\gamma_{S^{*}}(r, a c, v) \vee \gamma_{S}(v, d, s)\right] \\
& =\gamma_{S^{*}}(r, a c d, s)=\gamma_{S^{*}}(r, a b, s) .
\end{aligned}
$$

Hence the result is true for $|b|=k$. Thus completes the proof.

Proposition 4.3 If $M=(N, U, S)$ is an INFSSM, then

$$
\alpha_{S^{*}}(r, a, s)=\alpha_{S^{*}}(s, a, r), \beta_{S^{*}}(r, a, s)=\beta_{S^{*}}(s, a, r)
$$

and

$$
\gamma_{S^{*}}(r, a, s)=\gamma_{S^{*}}(s, a, r)
$$

for all $r, s \in N$ and $a \in U^{*}$.

Proof Let $r, s \in N$ and $a \in U^{*}$. We prove the result by induction on $|a|=k$. If $k=0$, then $b=\zeta$, hence

$$
\begin{aligned}
& \alpha_{S^{*}}(r, a, s)=\alpha_{S^{*}}(r, \zeta, s)=\alpha_{S^{*}}(s, \zeta, r)=\alpha_{S^{*}}(s, a, r), \\
& \beta_{S^{*}}(r, a, s)=\beta_{S^{*}}(r, \zeta, s)=\beta_{S^{*}}(s, \zeta, r)=\beta_{S^{*}}(s, a, r)
\end{aligned}
$$

and

$$
\gamma_{S^{*}}(r, a, s)=\gamma_{S^{*}}(r, \zeta, s)=\gamma_{S^{*}}(s, \zeta, r)=\gamma_{S^{*}}(s, a, r) .
$$

Therefore the result is true for $k=0$. Assume that the result is true for $|b|=k-1$. That is for all $b \in U^{*}$ with $|b|=k-1, k>0$, we have

$$
\alpha_{S^{*}}(r, b, s)=\alpha_{S^{*}}(s, b, r), \beta_{S^{*}}(r, b, s)=\beta_{S^{*}}(s, b, r)
$$

and

$$
\gamma_{S^{*}}(r, b, s)=\gamma_{S^{*}}(s, b, r) .
$$

Let $x \in U$ and $b \in U^{*}$ be such that $a=b x$. Then

$$
\begin{aligned}
\alpha_{S^{*}}(r, a, s) & =\alpha_{S^{*}}(r, b x, s)=\vee_{v \in N}\left[\alpha_{S^{*}}(r, b, v) \wedge \alpha_{S}(v, x, s)\right] \\
& =\vee_{v \in N}\left[\alpha_{S^{*}}(v, b, r) \wedge \alpha_{S}(s, x, r)\right] \\
& =\vee_{v \in N}\left[\alpha_{S^{*}}(v, b, r) \wedge \alpha_{S^{*}}(s, x, r)\right] \\
& =\vee_{v \in N}\left[\alpha_{S^{*}}(s, x, r) \wedge \alpha_{S^{*}}(r, b, v)\right] \\
& =\alpha_{S^{*}}(s, x b, r)=\alpha_{S^{*}}(s, b x, r)=\alpha_{S^{*}}(s, a, r), \\
\beta_{S^{*}}(r, a, s) & =\beta_{S^{*}}(r, b x, s)=\wedge_{v \in N}\left[\beta_{S^{*}}(r, b, v) \vee \beta_{S}(v, x, s)\right] \\
& =\wedge_{v \in N}\left[\beta_{S^{*}}(v, b, r) \vee \beta_{S}(s, x, r)\right] \\
& =\wedge_{v \in N}\left[\beta_{S^{*}}(v, b, r) \vee \beta_{S^{*}}(s, x, r)\right] \\
& =\wedge_{v \in N}\left[\beta_{S^{*}}(s, x, r) \vee \beta_{S^{*}}(r, b, v)\right] \\
& =\beta_{S^{*}}(s, x b, r)=\beta_{S^{*}}(s, b x, r)=\beta_{S^{*}}(s, a, r)
\end{aligned}
$$


and

$$
\begin{aligned}
\gamma_{S^{*}}(r, a, s) & =\gamma_{S^{*}}(r, b x, s)=\wedge_{v \in N}\left[\gamma_{S^{*}}(r, b, v) \vee \gamma_{S}(v, x, s)\right] \\
& =\wedge_{v \in N}\left[\gamma_{S^{*}}(v, b, r) \vee \gamma_{S}(s, x, r)\right] \\
& =\wedge_{v \in N}\left[\gamma_{S^{*}}(v, b, r) \vee \gamma_{S^{*}}(s, x, r)\right] \\
& =\wedge_{v \in N}\left[\gamma_{S^{*}}(s, x, r) \vee \gamma_{S^{*}}(r, b, v)\right] \\
& =\gamma_{S^{*}}(s, x b, r)=\gamma_{S^{*}}(s, b x, r)=\gamma_{S^{*}}(s, a, r) .
\end{aligned}
$$

This shows that the result is true for $|b|=k$.

Proposition 4.4 If $M=(N, U, S)$ is an INFSSM, then

$$
\alpha_{S^{*}}(r, a b, s)=\alpha_{S^{*}}(r, b a, s), \beta_{S^{*}}(r, a b, s)=\beta_{S^{*}}(r, b a, s)
$$

and

$$
\gamma_{S^{*}}(r, a b, s)=\gamma_{S^{*}}(r, b a, s)
$$

for all $r, s \in N$ and $a, b \in U^{*}$.

Proof Let $r, s \in N$ and $a, b \in U^{*}$. We prove the result by induction on $|b|=k$. If $k=0$, then $b=\zeta$, hence

$$
\begin{aligned}
& \alpha_{S^{*}}(r, a b, s)=\alpha_{S^{*}}(r, a \zeta, s)=\alpha_{S^{*}}(r, a, s)=\alpha_{S^{*}}(r, \zeta a, s)=\alpha_{S^{*}}(r, b a, s), \\
& \beta_{S^{*}}(r, a b, s)=\beta_{S^{*}}(r, a \zeta, s)=\beta_{S^{*}}(r, a, s)=\beta_{S^{*}}(r, \zeta a, s)=\beta_{S^{*}}(r, b a, s)
\end{aligned}
$$

and

$$
\gamma_{S^{*}}(r, a b, s)=\gamma_{S^{*}}(r, a \zeta, s)=\gamma_{S^{*}}(r, a, s)=\gamma_{S^{*}}(r, \zeta a, s)=\gamma_{S^{*}}(r, b a, s) .
$$

Therefore the result is true for $k=0$. Suppose that the result is true for $|c|=k-1$. That is for all $c \in U^{*}$ with $|c|=k-1, k>0$. Let $d \in U$ be such that $b=c d$. Then

$$
\begin{aligned}
\alpha_{S^{*}}(r, a b, s) & =\alpha_{S^{*}}(r, a c d, s)=\vee_{v \in N}\left[\alpha_{S^{*}}(r, a c, v) \wedge \alpha_{S}(v, d, s)\right] \\
& =\vee_{v \in N}\left[\alpha_{S^{*}}(r, c a, v) \wedge \alpha_{S}(v, d, s)\right] \\
& =\vee_{v \in N}\left[\alpha_{S^{*}}(v, c a, r) \wedge \alpha_{S}(s, d, v)\right] \\
& =\vee_{v \in N}\left[\alpha_{S}(s, d, v) \wedge \alpha_{S^{*}}(v, c a, r)\right] \\
& =\alpha_{S^{*}}(s, d c a, r)=\vee_{v \in N}\left[\alpha_{S^{*}}(s, d c, v) \wedge \alpha_{S^{*}}(v, a, r)\right] \\
& =\vee_{v \in N}\left[\alpha_{S^{*}}(s, c d, v) \wedge \alpha_{S^{*}}(v, a, r)\right]=\alpha_{S^{*}}(s, c d a, r) \\
& =\alpha_{S^{*}}(r, c d a, s)=\alpha_{S^{*}}(r, b a, s), \\
\beta_{S^{*}}(r, a b, s) & =\beta_{S^{*}}(r, a c d, s)=\wedge_{v \in N}\left[\beta_{S^{*}}(r, a c, v) \vee \beta_{S}(v, d, s)\right] \\
& =\wedge_{v \in N}\left[\beta_{S^{*}}(r, c a, v) \vee \beta_{S}(v, d, s)\right] \\
& =\wedge_{v \in N}\left[\beta_{S^{*}}(v, c a, r) \vee \beta_{S}(s, d, v)\right] \\
& =\wedge_{v \in N}\left[\beta_{S}(s, d, v) \vee \beta_{S^{*}}(v, c a, r)\right] \\
& =\beta_{S^{*}}(s, d c a, r)=\wedge_{v \in N}\left[\beta_{S^{*}}(s, d c, v) \vee \beta_{S^{*}}(v, a, r)\right] \\
& =\wedge_{v \in N}\left[\beta_{S^{*}}(s, c d, v) \vee \beta_{S^{*}}(v, a, r)\right]=\beta_{S^{*}}(s, c d a, r) \\
& =\beta_{S^{*}}(r, c d a, s)=\beta_{S^{*}}(r, b a, s)
\end{aligned}
$$


and

$$
\begin{aligned}
\gamma_{S^{*}}(r, a b, s) & =\gamma_{S^{*}}(r, a c d, s)=\wedge_{v \in N}\left[\gamma_{S^{*}}(r, a c, v) \vee \gamma_{S}(v, d, s)\right] \\
& =\wedge_{v \in N}\left[\gamma_{S^{*}}(r, c a, v) \vee \gamma_{S}(v, d, s)\right] \\
& =\wedge_{v \in N}\left[\gamma_{S^{*}}(v, c a, r) \vee \gamma_{S}(s, d, v)\right] \\
& =\wedge_{v \in N}\left[\gamma_{S}(s, d, v) \vee \gamma_{S^{*}}(v, c a, r)\right] \\
& =\gamma_{S^{*}}(s, d c a, r)=\wedge_{v \in N}\left[\gamma_{S^{*}}(s, d c, v) \vee \gamma_{S^{*}}(v, a, r)\right] \\
& =\wedge_{v \in N}\left[\gamma_{S^{*}}(s, c d, v) \vee \gamma_{S^{*}}(v, a, r)\right]=\gamma_{S^{*}}(s, c d a, r) \\
& =\gamma_{S^{*}}(r, c d a, s)=\gamma_{S^{*}}(r, b a, s) .
\end{aligned}
$$

This shows that the result is true for $|b|=k$.

Definition 4.5 Let $M_{S}=\left(N_{1}, U_{1}, S\right)$ and $M_{T}=\left(N_{2}, U_{2}, T\right)$ be two INFSMs. A pair $(\varphi, \psi)$ of mappings $\varphi: N_{1} \rightarrow N_{2}$ and $\psi: U_{1} \rightarrow U_{2}$ is called homomorphism, written as $(\varphi, \psi): M_{S} \rightarrow M_{T}$, if it satisfies:

$$
\alpha_{S}(r, a, s) \leq \alpha_{T}(\varphi(r), \psi(a), \varphi(s)), \beta_{S}(r, a, s) \geq \beta_{T}(\varphi(r), \psi(a), \varphi(s))
$$

and

$$
\gamma_{S}(r, a, s) \geq \gamma_{T}(\varphi(r), \psi(a), \varphi(s))
$$

for all $r, s \in N_{1}$ and $a \in U_{1}$.

Example 4.6 Let $M_{S}=\left(N_{1}, U_{1}, S\right)$ and $M_{T}=\left(N_{2}, U_{2}, T\right)$ be two INFSMs. Where $N_{1}=$ $\left\{s_{1}, s_{2}, s_{3}\right\}, U_{1}=\{x, y\}, N_{2}=\left\{n_{1}, n_{2}\right\}$ and $U_{2}=\{x, y\}, S$ and $T$ are defined below:

$$
\begin{aligned}
& \alpha_{S}\left(s_{1}, x, s_{1}\right)=[0.1,0.2], \quad \beta_{S}\left(s_{1}, x, s_{1}\right)=[0.6,0.7], \quad \gamma_{S}\left(s_{1}, x, s_{1}\right)=[0.8,0.9], \\
& \alpha_{S}\left(s_{1}, x, s_{2}\right)=[0.15,0.25], \quad \beta_{S}\left(s_{1}, x, s_{2}\right)=[0.55,0.65], \quad \gamma_{S}\left(s_{1}, x, s_{1}\right)=[0.75,0.85], \\
& \alpha_{S}\left(s_{1}, y, s_{2}\right)=[0.2,0.3], \quad \beta_{S}\left(s_{1}, y, s_{2}\right)=[0.5,0.6], \quad \gamma_{S}\left(s_{1}, y, s_{2}\right)=[0.7,0.8], \\
& \alpha_{S}\left(s_{2}, x, s_{1}\right)=[0.25,0.35], \quad \beta_{S}\left(s_{2}, x, s_{1}\right)=[0.4,0.5], \quad \gamma_{S}\left(s_{2}, x, s_{1}\right)=[0.65,0.75], \\
& \alpha_{S}\left(s_{2}, y, s_{3}\right)=[0.3,0.4], \quad \beta_{S}\left(s_{2}, y, s_{3}\right)=[0.35,0.45], \quad \gamma_{S}\left(s_{2}, y, s_{3}\right)=[0.6,0.7], \\
& \alpha_{S}\left(s_{3}, x, s_{3}\right)=[0.5,0.6], \quad \beta_{S}\left(s_{3}, x, s_{3}\right)=[0.3,0.35], \quad \gamma_{S}\left(s_{3}, x, s_{3}\right)=[0.55,0.65], \\
& \alpha_{S}\left(s_{3}, y, s_{2}\right)=[0.35,0.45], \quad \beta_{S}\left(s_{3}, y, s_{2}\right)=[0.3,0.4], \quad \gamma_{S}\left(s_{3}, y, s_{2}\right)=[0.5,0.6] .
\end{aligned}
$$

The transition daigram is given below:

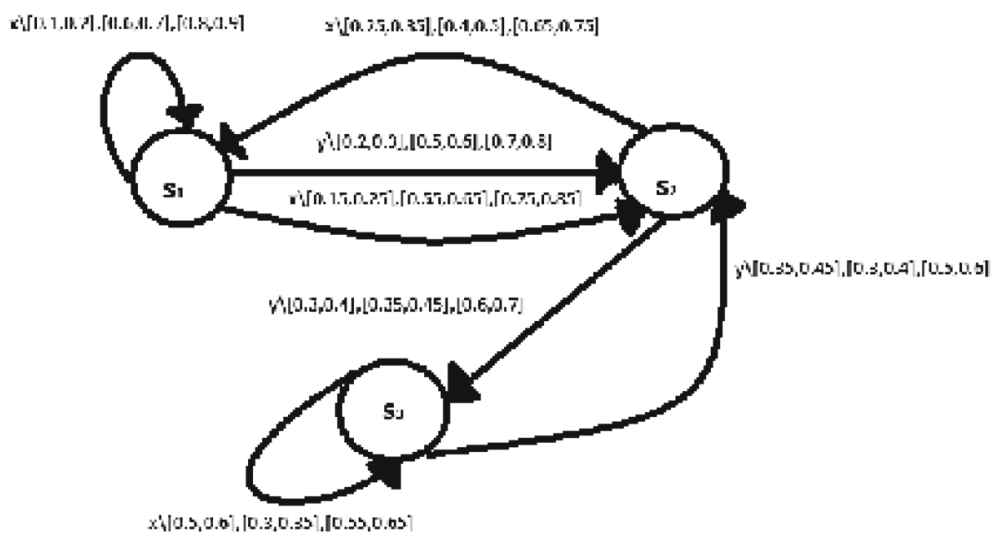


Define a mappings $\varphi: N_{1} \rightarrow N_{2}$ and $\psi: U_{1} \rightarrow U_{2}$, by $\varphi\left(s_{1}\right)=\varphi\left(s_{2}\right)=n_{1}, \varphi\left(s_{3}\right)=$ $n_{2}, \psi(x)=x$ and $\psi(y)=y$.

The homomorphic image of $M_{S}$

$\alpha_{T}\left(n_{1}, x, n_{1}\right)=[0.5,0.6], \quad \beta_{T}\left(n_{1}, x, n_{1}\right)=[0.3,0.4], \quad \gamma_{T}\left(n_{1}, x, n_{1}\right)=[0.5,0.6]$,

$\alpha_{T}\left(n_{1}, y, n_{1}\right)=[0.55,0.65], \quad \beta_{T}\left(n_{1}, y, n_{1}\right)=[0.29,0.39], \quad \gamma_{T}\left(n_{1}, y, n_{1}\right)=[0.4,0.45]$,

$\alpha_{T}\left(n_{1}, y, n_{2}\right)=[0.6,0.7], \quad \beta_{T}\left(n_{1}, y, n_{2}\right)=[0.21,0.2], \quad \gamma_{T}\left(n_{1}, y, n_{2}\right)=[0.35,0.42]$,

$\alpha_{T}\left(n_{2}, x, n_{2}\right)=[0.65,0.75], \quad \beta_{T}\left(n_{2}, x, n_{2}\right)=[0.2,0.25], \quad \gamma_{T}\left(n_{2}, x, n_{2}\right)=[0.3,0.35]$,

$\alpha_{T}\left(n_{2}, x, n_{1}\right)=[0.7,0.8], \quad \beta_{T}\left(n_{2}, x, n_{1}\right)=[0.15,0.2], \quad \gamma_{T}\left(n_{2}, x, n_{1}\right)=[0.25,0.3]$,

$\alpha_{T}\left(n_{2}, y, n_{1}\right)=[0.75,0.85], \quad \beta_{T}\left(n_{2}, y, n_{1}\right)=[0.1,0.15], \quad \gamma_{T}\left(n_{2}, y, n_{1}\right)=[0.1,0.2]$.

The

$x \backslash[0.5,0.6],[0.3,0.4],[0.5,0.6]$

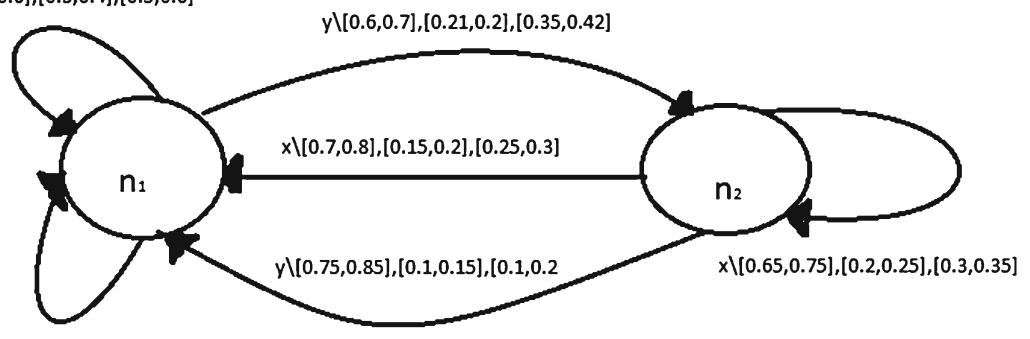

$y \backslash[0.55,0.65],[0.29,0.39],[0.4,0.45]$

Definition 4.7 Let $M_{S}=\left(N_{1}, U_{1}, S\right)$ and $M_{T}=\left(N_{2}, U_{2}, T\right)$ be two INFSMs. A pair $(\varphi, \psi)$ of mappings $\varphi: N_{1} \rightarrow N_{2}$ and $\psi: U_{1} \rightarrow U_{2}$ is called a strong homomorphism, written as $(\varphi, \psi): M_{S} \rightarrow M_{T}$, if it satisfies:

$$
\begin{aligned}
& \alpha_{T}(\varphi(r), \psi(a), \varphi(s))=\vee\left\{\alpha_{S}(r, a, v) \mid v \in N_{1}, \varphi(v)=\varphi(s)\right\} \\
& \beta_{T}(\varphi(r), \psi(a), \varphi(s))=\wedge\left\{\beta_{S}(r, a, v) \mid v \in N_{1}, \varphi(v)=\varphi(s)\right\}
\end{aligned}
$$

and

$$
\gamma_{T}(\varphi(r), \psi(a), \varphi(s))=\wedge\left\{\gamma_{S}(r, a, v) \mid v \in N_{1}, \varphi(v)=\varphi(s)\right\}
$$

for all $r, s \in N_{1}$ and $a \in U_{1}$. If $U_{1}=U_{2}$ and $\psi$ is the identity map, then we simply write $\varphi: M_{S} \rightarrow M_{T}$ and say that $\varphi$ is a homomorphism or strong homomorphism accordingly. If $(\varphi, \psi)$ is a strong homorphism with $\varphi$ is one-one, then

$$
\alpha_{T}(\varphi(r), \psi(a), \varphi(s))=\alpha_{S}(r, a, s), \beta_{T}(\varphi(r), \psi(a), \varphi(s))=\beta_{S}(r, a, s)
$$

and

$$
\gamma_{T}(\varphi(r), \psi(a), \varphi(s))=\gamma_{S}(r, a, s)
$$

for all $r, s \in N_{1}$ and $a \in U_{1}$.

Theorem 4.8 Let $M_{S}=\left(N_{1}, U_{1}, S\right)$ and $M_{T}=\left(N_{2}, U_{2}, T\right)$ be two INFSMs. Let $(\varphi, \psi): M_{S} \rightarrow M_{T}$ be an onto strong homomorphism. If $M_{S}$ is a commutative, then so is $M_{T}$. 
Proof Let $r_{2}, s_{2} \in N_{2}$. Then there are $r_{1}, s_{1} \in N_{1}$ such that $\varphi\left(r_{1}\right)=r_{2}$ and $\varphi\left(s_{1}\right)=s_{2}$. Let $x_{2}, y_{2} \in U_{2}$. Then there exists $x_{1}, y_{1} \in U_{1}$ such that $\psi\left(x_{1}\right)=x_{2}$ and $\psi\left(y_{1}\right)=y_{2}$. Since $M_{S}$ is commutative, we have

$$
\begin{aligned}
\alpha_{T^{*}}\left(r_{2}, x_{2} y_{2}, s_{2}\right) & =\alpha_{T^{*}}\left(\varphi\left(r_{1}\right), \psi\left(x_{1}\right) \psi\left(y_{1}\right), \varphi\left(s_{1}\right)\right) \\
& =\alpha_{T^{*}}\left(\varphi\left(r_{1}\right), \psi\left(x_{1}, y_{1}\right), \varphi\left(s_{1}\right)\right) \\
& =\vee\left\{\alpha_{S^{*}}\left(r_{1}, x_{1} y_{1}, v_{1}\right) \mid v_{1} \in N_{1}, \varphi\left(v_{1}\right)=\varphi\left(s_{1}\right)\right\} \\
& =\vee\left\{\alpha_{S^{*}}\left(r_{1}, y_{1} x_{1}, v_{1}\right) \mid v_{1} \in N_{1}, \varphi\left(v_{1}\right)=\varphi\left(s_{1}\right)\right\} \\
& =\alpha_{T^{*}}\left(\varphi\left(r_{1}\right), \psi\left(y_{1} x_{1}\right), \varphi\left(s_{1}\right)\right) \\
& =\alpha_{T^{*}}\left(r_{2}, y_{2} x_{2}, s_{2}\right), \\
\beta_{T^{*}}\left(r_{2}, x_{2} y_{2}, s_{2}\right) & =\beta_{T^{*}}\left(\varphi\left(r_{1}\right), \psi\left(x_{1}\right) \psi\left(y_{1}\right), \varphi\left(s_{1}\right)\right) \\
& =\beta_{T^{*}}\left(\varphi\left(r_{1}\right), \psi\left(x_{1}, y_{1}\right), \varphi\left(s_{1}\right)\right) \\
& =\wedge\left\{\beta_{S^{*}}\left(r_{1}, x_{1} y_{1}, v_{1}\right) \mid v_{1} \in N_{1}, \varphi\left(v_{1}\right)=\varphi\left(s_{1}\right)\right\} \\
& =\wedge\left\{\beta_{S^{*}}\left(r_{1}, y_{1} x_{1}, v_{1}\right) \mid v_{1} \in N_{1}, \varphi\left(v_{1}\right)=\varphi\left(s_{1}\right)\right\} \\
& =\beta_{T^{*}}\left(\varphi\left(r_{1}\right), \psi\left(y_{1} x_{1}\right), \varphi\left(s_{1}\right)\right) \\
& =\beta_{T^{*}}\left(\varphi\left(r_{1}\right), \psi\left(y_{1}\right) \psi\left(x_{1}\right), \varphi\left(s_{1}\right)\right) \\
& =\beta_{T^{*}}\left(r_{2}, y_{2} x_{2}, s_{2}\right)
\end{aligned}
$$

and

$$
\begin{aligned}
\gamma_{T^{*}}\left(r_{2}, x_{2} y_{2}, s_{2}\right) & =\gamma_{T^{*}}\left(\varphi\left(r_{1}\right), \psi\left(x_{1}\right) \psi\left(y_{1}\right), \varphi\left(s_{1}\right)\right) \\
& =\gamma_{T^{*}}\left(\varphi\left(r_{1}\right), \psi\left(x_{1}, y_{1}\right), \varphi\left(s_{1}\right)\right) \\
& =\wedge\left\{\gamma_{S^{*}}\left(r_{1}, x_{1} y_{1}, v_{1}\right) \mid v_{1} \in N_{1}, \varphi\left(v_{1}\right)=\varphi\left(s_{1}\right)\right\} \\
& =\wedge\left\{\gamma_{S^{*}}\left(r_{1}, y_{1} x_{1}, v_{1}\right) \mid v_{1} \in N_{1}, \varphi\left(v_{1}\right)=\varphi\left(s_{1}\right)\right\} \\
& =\gamma_{T^{*}}\left(\varphi\left(r_{1}\right), \psi\left(y_{1} x_{1}\right), \varphi\left(s_{1}\right)\right) \\
& =\gamma_{T^{*}}\left(\varphi\left(r_{1}\right), \psi\left(y_{1}\right) \psi\left(x_{1}\right), \varphi\left(s_{1}\right)\right) \\
& =\gamma_{T^{*}}\left(r_{2}, y_{2} x_{2}, s_{2}\right) .
\end{aligned}
$$

Hence $M_{T}$ is a commutative INFSM. This completes the proof.

Proposition 4.9 Let $M_{S}=\left(N_{1}, U_{1}, S\right)$ and $M_{T}=\left(N_{2}, U_{2}, T\right)$ be two INFSMs. Let $(\varphi, \psi): M_{S} \rightarrow M_{T}$ be a strong homomorphism. Then

$$
\begin{gathered}
\left(\forall u, v \in N_{1}\right)\left(\forall a \in U_{1}\right)\left(\alpha_{T}(\varphi(u), \psi(a), \varphi(v))>[0,0]\right. \\
\Rightarrow\left(\exists w \in N_{1}\right)\left(\alpha_{S}(u, a, v)>[0,0], \varphi(w)=\varphi(v)\right), \\
\left(\forall u, v \in N_{1}\right)\left(\forall a \in U_{1}\right)\left(\beta_{T}(\varphi(u), \psi(a), \varphi(v))<[1,1]\right. \\
\Rightarrow\left(\exists w \in N_{1}\right)\left(\beta_{S}(u, a, v)<[1,1], \varphi(w)=\varphi(v)\right),
\end{gathered}
$$

and

$$
\begin{gathered}
\left(\forall u, v \in N_{1}\right)\left(\forall a \in U_{1}\right)\left(\gamma_{T}(\varphi(u), \psi(a), \varphi(v))<[1,1]\right. \\
\Rightarrow\left(\exists w \in N_{1}\right)\left(\gamma_{S}(u, a, v)<[1,1], \varphi(w)=\varphi(v)\right) .
\end{gathered}
$$

\section{Moreover,}

$$
\begin{aligned}
\left(\forall z \in N_{1}\right)(\varphi(z) & =\varphi(u) \Rightarrow \alpha_{S}(u, a, w) \\
& \geq \alpha_{S}(z, a, r), \beta_{S}(u, a, w) \leq \beta_{S}(z, a, r) \text { and } \gamma_{S}(u, a, w) \leq \gamma_{S}(z, a, r) .
\end{aligned}
$$


Proof Let $u, v, z \in N_{1}$ and $a \in U_{1}$. Assume that $\alpha_{T}(\varphi(u), \psi(a), \varphi(v))>[0,0]$, $\left(\beta_{T}(\varphi(u), \psi(a), \varphi(v))<[1,1]\right.$ and $\left(\gamma_{T}(\varphi(u), \psi(a), \varphi(v))<[1,1]\right.$. Then

$$
\begin{aligned}
& \vee\left\{\alpha_{S}\left(u, a, v_{1}\right) \mid v_{1} \in N_{1}, \varphi\left(v_{1}\right)=\varphi(v)\right\}>[0,0], \\
& \wedge\left\{\beta_{S}\left(u, a, v_{1}\right) \mid v_{1} \in N_{1}, \varphi\left(v_{1}\right)=\varphi(v)\right\}<[1,1]
\end{aligned}
$$

and

$$
\wedge\left\{\beta_{S}\left(u, a, v_{1}\right) \mid v_{1} \in N_{1}, \varphi\left(v_{1}\right)=\varphi(v)\right\}<[1,1] .
$$

Since $N_{1}$ is finite, it follows that there exists $w \in N_{1}$ such that $\varphi(w)=\varphi(v)$,

$$
\begin{aligned}
\alpha_{S}(u, a, w) & =\vee\left\{\alpha_{S}\left(u, a, v_{1}\right) \mid v_{1} \in N_{1}, \varphi\left(v_{1}\right)=\varphi(w)\right\}>[0,0], \\
\beta_{S}(u, a, v) & =\wedge\left\{\beta_{S}\left(u, a, v_{1}\right) \mid v_{1} \in N_{1}, \varphi\left(v_{1}\right)=\varphi(w)\right\}<[1,1]
\end{aligned}
$$

and

$$
\gamma_{S}(u, a, v)=\wedge\left\{\gamma_{S}\left(u, a, v_{1}\right) \mid v_{1} \in N_{1}, \varphi\left(v_{1}\right)=\varphi(w)\right\}<[1,1] .
$$

Now suppose that $\varphi(z)=\varphi(u)$ for every $z \in N_{1}$. Then

$$
\begin{aligned}
\alpha_{S}(u, a, w) & =\alpha_{T}(\varphi(u), \psi(a), \varphi(v))=\alpha_{T}(\varphi(z), \psi(a), \varphi(v)) \\
& =\vee\left\{\alpha_{S}\left(z, a, v_{1}\right) \mid v_{1} \in N_{1}, \varphi\left(v_{1}\right)=\varphi(v)\right\} \geq \alpha_{S}(z, a, v), \\
\beta_{S}(u, a, w) & =\beta_{T}(\varphi(u), \psi(a), \varphi(v))=\beta_{T}(\varphi(z), \psi(a), \varphi(v)) \\
& =\wedge\left\{\beta_{S}\left(z, a, v_{1}\right) \mid v_{1} \in N_{1}, \varphi\left(v_{1}\right)=\varphi(v)\right\} \leq \beta_{S}(z, a, v)
\end{aligned}
$$

and

$$
\begin{aligned}
\gamma_{S}(u, a, w) & =\gamma_{T}(\varphi(u), \psi(a), \varphi(v))=\gamma_{T}(\varphi(z), \psi(a), \varphi(v)) \\
& =\wedge\left\{\gamma_{S}\left(z, a, v_{1}\right) \mid v_{1} \in N_{1}, \varphi\left(v_{1}\right)=\varphi(v)\right\} \leq \gamma_{S}(z, a, v)
\end{aligned}
$$

which is the required proof.

Lemma 4.10 Let $M_{S}=\left(N_{1}, U_{1}, S\right)$ and $M_{T}=\left(N_{2}, U_{2}, T\right)$ be two INFSMs. Let $(\varphi, \psi): M_{S} \rightarrow M_{T}$ be a homomorphism. Define a mapping $\psi^{*}: U_{1}^{*} \rightarrow U_{2}^{*}$ by $\psi^{*}(\zeta)=\zeta$ and $\psi^{*}(x y)=\psi^{*}(x) \psi^{*}(y)$ for all $x \in U_{1}^{*}$ and $y \in U_{1}$. Then $\psi^{*}(a b)=\psi^{*}(a) \psi^{*}(b)$ for all $a, b \in U_{1}^{*}$.

Proof Let $a, b \in U_{1}^{*}$. We prove the result by induction on $|b|=k$. If $k=0$, then $b=\zeta$. Therefore $a b=a \zeta=a$. Hence

$$
\psi^{*}(a b)=\psi^{*}(a)=\psi^{*}(a) \zeta=\psi^{*}(a) \psi^{*}(\zeta)=\psi^{*}(a) \psi^{*}(b)
$$

which shows that the result is true for $k=0$. Let us assume that the result is true for each $c \in U_{1}^{*}$ such that $|c|=k-1$. That is

$$
\psi^{*}(a b)=\psi^{*}(a) \psi^{*}(b) .
$$

Let $b=c d$, where $c \in U_{1}^{*}$ and $d \in U_{1}$ be such that $|c|=k-1, k>0$. Then $\psi^{*}(a b)=\psi^{*}(a c d)=\psi^{*}(a c) \psi(d)=\psi^{*}(a) \psi^{*}(c) \psi(d)=\psi^{*}(a) \psi^{*}(c d)=\psi^{*}(a) \psi^{*}(b)$. Therefore the result is true for $|b|=k$. 
Theorem 4.11 Let $M_{S}=\left(N_{1}, U_{1}, S\right)$ and $M_{T}=\left(N_{2}, U_{2}, T\right)$ be two INFSMs. Let $(\varphi, \psi): M_{S} \rightarrow M_{T}$ be a homomorphism. Then

$$
\alpha_{S^{*}}(r, a, s) \leq \alpha_{T^{*}}\left(\varphi(r), \psi^{*}(a), \varphi(s)\right), \beta_{S^{*}}(r, a, s) \geq \beta_{T^{*}}\left(\varphi(r), \psi^{*}(a), \varphi(s)\right)
$$

and

$$
\gamma_{S^{*}}(r, a, s) \geq \gamma_{T^{*}}\left(\varphi(r), \psi^{*}(a), \varphi(s)\right)
$$

for all $r, s \in N_{1}$ and $a \in U_{1}^{*}$.

Proof Let $r, s \in N_{1}$ and $a \in U_{1}^{*}$. We prove the result by induction on $|a|=k$. If $k=0$, then $a=\zeta$ and so $\psi^{*}(a)=\psi^{*}(\zeta)=\zeta$. If $r=s$, then

$$
\begin{aligned}
& \alpha_{S^{*}}(r, a, s)=\alpha_{S^{*}}(r, \zeta, s)=[1,1]=\alpha_{T^{*}}(\varphi(r), \zeta, \varphi(s))=\alpha_{T^{*}}\left(\varphi(r), \psi^{*}(a), \varphi(s)\right), \\
& \beta_{S^{*}}(r, a, s)=\beta_{S^{*}}(r, \zeta, s)=[0,0]=\beta_{T^{*}}(\varphi(r), \zeta, \varphi(s))=\beta_{T^{*}}\left(\varphi(r), \psi^{*}(a), \varphi(s)\right)
\end{aligned}
$$

and

$$
\gamma_{S^{*}}(r, a, s)=\gamma_{S^{*}}(r, \zeta, s)=[0,0]=\gamma_{T^{*}}(\varphi(r), \zeta, \varphi(s))=\gamma_{T^{*}}\left(\varphi(r), \psi^{*}(a), \varphi(s)\right) .
$$

If $r \neq s$, then

$$
\begin{aligned}
& \alpha_{S^{*}}(r, a, s)=\alpha_{S^{*}}(r, \zeta, s)=[0,0] \leq \alpha_{T^{*}}\left(\varphi(r), \psi^{*}(a), \varphi(s)\right), \\
& \beta_{S^{*}}(r, a, s)=\beta_{S^{*}}(r, \zeta, s)=[1,1] \geq \beta_{T^{*}}\left(\varphi(r), \psi^{*}(a), \varphi(s)\right)
\end{aligned}
$$

and

$$
\gamma_{S^{*}}(r, a, s)=\gamma_{S^{*}}(r, \zeta, s)=[1,1] \geq \gamma_{T^{*}}\left(\varphi(r), \psi^{*}(a), \varphi(s)\right)
$$

Therefore the result is true for $k=0$. Let us assume that the result is true for all $b \in U_{1}^{*}$ such that $|b|=k-1, k>0$. Let $a=b c$, where $b \in U_{1}^{*}, c \in U_{1}$ and $|b|=k-1$. Then

$$
\begin{aligned}
\alpha_{S^{*}}(r, a, s) & =\alpha_{S^{*}}(r, b c, s)=\vee_{v \in N_{1}}\left[\alpha_{S^{*}}(r, b, v) \wedge \alpha_{S^{*}}(v, c, s)\right] \\
& \leq \vee_{v \in N_{1}}\left[\alpha_{T^{*}}\left(\varphi(r), \psi^{*}(b), \varphi(v)\right) \wedge \alpha_{T^{*}}(\varphi(v), \psi(c), \varphi(s))\right] \\
& \leq \vee_{v^{\circ} \in N_{1}}\left[\alpha_{T^{*}}\left(\varphi(r), \psi^{*}(b), v^{\circ}\right) \wedge \alpha_{T^{*}}\left(v^{\circ}, \psi(c), \varphi(s)\right)\right] \\
& =\alpha_{T^{*}}\left(\varphi(r), \psi^{*}(b) \psi(c), \varphi(s)\right) \\
& =\alpha_{T^{*}}\left(\varphi(r), \psi^{*}(b c), \varphi(s)\right) \\
& =\alpha_{T^{*}}\left(\varphi(r), \psi^{*}(a), \varphi(s)\right), \\
\beta_{S^{*}}(r, a, s) & =\beta_{S^{*}}(r, b c, s)=\wedge_{v \in N_{1}}\left[\beta_{S^{*}}(r, b, v) \vee \beta_{S^{*}}(v, c, s)\right] \\
& \geq \wedge_{v \in N_{1}}\left[\beta_{T^{*}}\left(\varphi(r), \psi^{*}(b), \varphi(v)\right) \vee \beta_{T^{*}}(\varphi(v), \psi(c), \varphi(s))\right] \\
& \geq \wedge_{v^{\circ} \in N_{1}}\left[\beta_{T^{*}}\left(\varphi(r), \psi^{*}(b), v^{\circ}\right) \vee \beta_{T^{*}}\left(v^{\circ}, \psi(c), \varphi(s)\right)\right] \\
& =\beta_{T^{*}}\left(\varphi(r), \psi^{*}(b) \psi(c), \varphi(s)\right) \\
& =\beta_{T^{*}}\left(\varphi(r), \psi^{*}(b c), \varphi(s)\right) \\
& =\beta_{T^{*}}\left(\varphi(r), \psi^{*}(a), \varphi(s)\right)
\end{aligned}
$$


and

$$
\begin{aligned}
\gamma_{S^{*}}(r, a, s) & =\gamma_{S^{*}}(r, b c, s)=\wedge_{v \in N_{1}}\left[\gamma_{S^{*}}(r, b, v) \vee \gamma_{S^{*}}(v, c, s)\right] \\
& \geq \wedge_{v \in N_{1}}\left[\gamma_{T^{*}}\left(\varphi(r), \psi^{*}(b), \varphi(v)\right) \vee \gamma_{T^{*}}(\varphi(v), \psi(c), \varphi(s))\right] \\
& \geq \wedge_{v^{\circ} \in N_{1}}\left[\gamma_{T^{*}}\left(\varphi(r), \psi^{*}(b), v^{\circ}\right) \vee \gamma_{T^{*}}\left(v^{\circ}, \psi(c), \varphi(s)\right)\right] \\
& =\gamma_{T^{*}}\left(\varphi(r), \psi^{*}(b) \psi(c), \varphi(s)\right) \\
& =\gamma_{T^{*}}\left(\varphi(r), \psi^{*}(b c), \varphi(s)\right) \\
& =\gamma_{T^{*}}\left(\varphi(r), \psi^{*}(a), \varphi(s)\right)
\end{aligned}
$$

which is the required proof.

Theorem 4.12 Let $M_{S}=\left(N_{1}, U_{1}, S\right)$ and $M_{T}=\left(N_{2}, U_{2}, T\right)$ be two INFSMs. Let $(\varphi, \psi): M_{S} \rightarrow M_{T}$ be a strong homomorphism. If $\varphi$ is one-one, then

$$
\alpha_{S^{*}}(r, a, s)=\alpha_{T^{*}}\left(\varphi(r), \psi^{*}(a), \varphi(s)\right), \quad \beta_{S^{*}}(r, a, s)=\beta_{T^{*}}\left(\varphi(r), \psi^{*}(a), \varphi(s)\right)
$$

and

$$
\gamma_{S^{*}}(r, a, s)=\gamma_{T^{*}}\left(\varphi(r), \psi^{*}(a), \varphi(s)\right)
$$

for all $r, s \in N_{1}$ and $a \in U_{1}^{*}$.

Proof Let us assume that $\varphi$ is $1-1$ and for $r, s \in N_{1}$ and $a \in U_{1}^{*}$. Let $|a|=k$. We prove the result by induction on on $|a|=k$. If $k=0$, then $a=\zeta$ and $\psi^{*}(\zeta)=\zeta$. Since $\varphi(r)=\varphi(s)$ if and only if $r=s$, we get

$$
\alpha_{S^{*}}(r, a, s)=\alpha_{S^{*}}(r, \zeta, s)=[1,1]
$$

if and only if

$$
\begin{aligned}
\alpha_{T^{*}}\left(\varphi(r), \psi^{*}(a), \varphi(s)\right) & =\alpha_{T^{*}}\left(\varphi(r), \psi^{*}(\zeta), \varphi(s)\right)=[1,1], \\
\beta_{S^{*}}(r, a, s) & =\beta_{S^{*}}(r, \zeta, s)=[0,0]
\end{aligned}
$$

if and only if

$$
\beta_{T^{*}}\left(\varphi(r), \psi^{*}(a), \varphi(s)\right)=\beta_{T^{*}}\left(\varphi(r), \psi^{*}(\zeta), \varphi(s)\right)=[0,0]
$$

and

$$
\gamma_{S^{*}}(r, a, s)=\gamma_{S^{*}}(r, \zeta, s)=[0,0]
$$

if and only if

$$
\gamma_{T^{*}}\left(\varphi(r), \psi^{*}(a), \varphi(s)\right)=\gamma_{T^{*}}\left(\varphi(r), \psi^{*}(\zeta), \varphi(s)\right)=[0,0] .
$$


Let us assume that the result is true for all $b \in U_{1}^{*}$ such that $|b|=k-1, k>0$. Let $a=b c$, where $|b|=k-1, k>0$ and $b \in U_{1}^{*}, c \in U_{1}$. Then

$$
\begin{aligned}
\alpha_{T^{*}}\left(\varphi(r), \psi^{*}(a), \varphi(s)\right) & =\alpha_{T^{*}}\left(\varphi(r), \psi^{*}(b c), \varphi(s)\right)=\alpha_{T^{*}}\left(\varphi(r), \psi^{*}(b) \psi(c), \varphi(s)\right) \\
& =\vee_{v \in N_{1}}\left[\alpha_{T^{*}}\left(\varphi(r), \psi^{*}(b), \varphi(v)\right) \wedge \alpha_{T}(\varphi(v), \psi(c), \varphi(s))\right] \\
& =\vee_{v \in N_{1}}\left[\alpha_{S^{*}}(r, b, v) \wedge \alpha_{S}(v, c, s)\right] \\
& =\alpha_{S^{*}}(r, b c, s)=\alpha_{S^{*}}(r, a, s), \\
\beta_{T^{*}}\left(\varphi(r), \psi^{*}(a), \varphi(s)\right) & =\beta_{T^{*}}\left(\varphi(r), \psi^{*}(b c), \varphi(s)\right)=\beta_{T^{*}}\left(\varphi(r), \psi^{*}(b) \psi(c), \varphi(s)\right) \\
& =\wedge_{v \in N_{1}}\left[\beta_{T^{*}}\left(\varphi(r), \psi^{*}(b), \varphi(v)\right) \vee \beta_{T}(\varphi(v), \psi(c), \varphi(s))\right] \\
& =\wedge_{v \in N_{1}}\left[\beta_{S^{*}}(r, b, v) \vee \beta_{S}(v, c, s)\right] \\
& =\beta_{S^{*}}(r, b c, s)=\beta_{S^{*}}(r, a, s)
\end{aligned}
$$

and

$$
\begin{aligned}
\gamma_{T^{*}}\left(\varphi(r), \psi^{*}(a), \varphi(s)\right) & =\gamma_{T^{*}}\left(\varphi(r), \psi^{*}(b c), \varphi(s)\right)=\gamma_{T^{*}}\left(\varphi(r), \psi^{*}(b) \psi(c), \varphi(s)\right) \\
& =\wedge_{v \in N_{1}}\left[\gamma_{T^{*}}\left(\varphi(r), \psi^{*}(b), \varphi(v)\right) \vee \gamma_{T}(\varphi(v), \psi(c), \varphi(s))\right] \\
& =\wedge_{v \in N_{1}}\left[\gamma_{S^{*}}(r, b, v) \vee \gamma_{S}(v, c, s)\right] \\
& =\gamma_{S^{*}}(r, b c, s)=\gamma_{S^{*}}(r, a, s)
\end{aligned}
$$

which is the required proof.

\section{Conclusion}

Using interval neutrosophic set we introduced the concept of interval neutrosophic finite state machine which is extension of fuzzy finite state machine and intuitionistic fuzzy finite state machine and discussed some related results. We also introduce the concept of interval neutrosophic switchboard state machine, homomorphism and storng homomorphism in interval neutrosophic finite state machine and discussed some related resuls. In future we work on interval neutrosophic automata.

\section{References}

1. Atanassov, K.T.: Intuitionistic fuzzy sets. Fuzzy Sets Syst. 20(1), 87-96 (1986)

2. Atanassov, K., Gargov, G.: Interval valued intuitionistic fuzzy sets. Fuzzy Sets Syst. 31(3), 343-349 (1989)

3. Gau, W.L., Buehrer, D.J.: Vague sets. IEEE Trans. Syst. Man Cybern. 23(2), 610-614 (1993)

4. Jun, Y.B.: Intuitionistic fuzzy finite state machines. J. Appl. Math. Comput. 17(1-2), 109-120 (2005)

5. Jun, Y.B.: Intuitionistic fuzzy finite switchboard state machines. J. Appl. Math. Comput. 20(1-2), 315-325 (2006)

6. Jun, Y.B., Kim, C.S., Yang, K.O.: Cubic sets. Ann. Fuzzy Math. Inform. 4(1), 83-98 (2012)

7. Kumbhojkar, H.V., Chaudhari, S.R.: On covering of products of fuzzy finite state machines. Fuzzy Sets Syst. 125(2), 215-222 (2002)

8. Lee, K. M.: Bipolar-valued fuzzy sets and their operations. In: Proc. Int. Conf. on Intelligent Technologies, Bangkok, Thailand, pp. 307-312 (2000)

9. Malik, D.S., Mordeson, J.N., Sen, M.K.: On subsystems of a fuzzy finite state machine. Fuzzy Sets Syst. 68(1), 83-92 (1994)

10. Malik, D.S., Mordeson, J.N., Sen, M.K.: Submachines of fuzzy finite state machines. J. Fuzzy Math. 2(4), 781-792 (1994)

11. Malik, D.S., Mordeson, J.N., Sen, M.K.: Products of fuzzy finite state machines. Fuzzy Sets Syst. 92(1), 95-102 (1997)

12. Smarandache, F.: Neutrosophic Probability, Set, and Logic, p. 105. Am. Res. Press, Rehoboth (1998) 
13. Smarandache, F.: A Unifying Field in Logics. Neutrosophy: Neutrosophic Probability, Set and Logic, pp. 1-141. American Research Press, Rehobothm, MA (1999)

14. Sato, Y., Kuroki, N.: Fuzzy finite switchboard state machines. J. Fuzzy Math. 10(4), 863-874 (2002)

15. Wang, H., Smarandache, F., Zhang, Y.Q., Sunderraman, R.: Interval Neutrosophic Sets and Logic: Theory and Applications in Computing, vol. 5. Hexis, Phoenix, AZ (2005)

16. Wang, H., Smarandache, F., Zhang, Y.Q., Sunderraman, R.: Single valued neutrosophic sets. Multispace Multistruct 4, 410-413 (2010)

17. Zadeh, L.A.: Information and control. Fuzzy Sets 8(3), 338-353 (1965)

18. Zadeh, L.A.: The Concept of a Linguistic Variable and its Application to Approximate Reasoning. Springer, US (1974) 